$F$ s, the role that $F$ plays within that theory, and the fact that $F$ correlates with $E$. Goldman cannot retort that the learner cannot learn about $F$ s because having the concept of $F$ presupposes the ability to distinguish $F$ s from non- $F$, as this would just beg the question against a theory-based account of concepts.

Finally, it is worrying that Goldman's argument make no appeal to special properties of folk psychological concepts. If this form of argument were valid, we could conclude that no concepts are theory-based. The argument would be that for all concepts there must be some category representation (CR) which is activated when concept instances are present and not otherwise, and this will simply not be possible for theoretical terms. The conclusion that people cannot have the concepts "proton," "gene," or "force" is counterintuitive enough to provide a reductio of Goldman's argument, if one were needed.

\title{
Nick Chater
}

Department of Psychology, University of Edinburgh, Edinburgh EH8 9JZ, Scottand

Electrontc mail: nicholas@cogsci.ed.ac.uk

[Gol] Goldman's argument against a functionalist or theorybased account of folk psychological terms is, I shall argue, both question-begging and fallacious. It is question-begging because Goldman begins by assuming, without argument, a categorizational view of concepts to the effect that to have a concept is to have an internal state (what Goldman calls a CR) which is active just when concept instances are present. He then argues that this assumption is incompatible with a theory-based view of concepts, according to which having a concept involves having an entire theory of the relevant domain. This is because the theoretical properties of a concept instance will generally not be available to the categorization system. An argument parallel to Goldman's, however, could start by assuming that concepts are defined in terms of theories and argue that, since it is not possible to distinguish instances from noninstances of a concept according to their theoretical properties, the categorizational account cannot be correct. Both arguments beg the question of whether a categorizational or theory-based view is more appropriate.

The argument is fallacious because, in any case, Goldman does not establish that the categorizational and theory-based views are incompatible. To see this, consider how Goldman's argument fares with a concept like mass, which is, after all, a paradigmatically theoretical term, connected with force, acceleration, gravitational laws, and so on. An object can be classified as having a certain mass purely in virtue of visual or tactile perceptual input, without any knowledge of causes or effects of that object which hold in virtue of that mass (Goldman's first difficulty); without any knowledge of the relevant subjunctive properties of the object, such as how it would move if various forces were applied (Coldman's second difficulty); and without knowing the type identity (i.e., category) of theoretically relevant properties, such as the forces acting on the object, and thus being sucked into a classificatory regress (Goldman's third difficulty). None of these problems arise, because classification is effected by detecting perceptual correlates of mass, rather than its constituent properties.

Goldman recognizes this possible rejoinder. He notes that a cube of sugar could be recognized as sugar by its whiteness, hardness, granularity rather than its theoretical properties such as solubility. More generally, a theoretical property $F$ can be detected by its correlated perceptible property $E$. Having recognized this possible way out, Goldman then gives a very puzzling argument against it. He claims that this correlation could only be learnt in the first place given that the learner has some independent way of recognizing $F s$, thus bringing back the original problem. This argument assumes that the learning of a correlation must occur by induction from observed $E, F$ pairs, but this is a very limited view of how learning can occur. For example, the learner could simply be told the theory relevant to 